\title{
Effectiveness of Metanx Prescription Medical Food on Small Nerve Fibers and Monofilament Sensation in Patients with Diabetic Peripheral Polyneuropathy
}

\author{
Victor F. McNamara1, Aaron I. Vinik², Lori Barrentine³, Edward B. De Vol ${ }^{4}$ \\ ${ }^{1}$ Central Florida Foot \& Ankle Specialists, PA, Orlando, FL, USA \\ ${ }^{2}$ Eastern Virginia Medical School, Norfolk, VA, USA \\ ${ }^{3}$ Nestlé Health Science-Pamlab, Inc., Covington, LA, USA \\ ${ }^{4} \mathrm{D} \&$ D Statistical Consultants, Dallas, TX, USA \\ Email: Ibarrentine@pamlab.com
}

Received 25 February 2016; accepted 3 May 2016; published 6 May 2016

Copyright (C) 2016 by authors and Scientific Research Publishing Inc.

This work is licensed under the Creative Commons Attribution International License (CC BY). http://creativecommons.org/licenses/by/4.0/

(c) (7) Open Access

\begin{abstract}
Purpose: Diabetic peripheral neuropathy (DPN) is prevalent among the population with type 2 diabetes, and treatment approaches are limited. The combination of L-methylfolate-methylcobalamin-pyridoxal-5-phospate (LMF-MC-PP, Metanx ${ }^{\circledR}$ ) is a prescription medical food that has demonstrated significant improvements in sensory perception and quality of life as well as reduced neuropathic pain in patients with DPN. The present study examined the effects of LMF-MC-PP on sensory perception and epidermal nerve fiber density (ENFD) among patients with confirmed DPN. Methods: Patients with type 2 diabetes and diagnosed with diabetic peripheral polyneuropathy, based on loss of vibratory perception, warm-cold discrimination or monofilament sensation, underwent bilateral lower extremity ENFD assessments via skin punch biopsy and were started on LMF-MC-PP. ENFD and monofilament testing were repeated at 6 months. Findings: Of 123 patients evaluated, all had monofilament testing at baseline and 6 months and 122 had assessments at both time points. A significant $(p<0.05)$ improvement in ENFD from baseline to 6 months was observed. A significant $(p=0.0001)$ improvement in monofilament testing also was observed. Overall, more patients had intact sensation after 6 months, with $60(48.8 \%)$ of 123 patients having positive monofilament test at baseline as compared to $95(77.2 \%)$ positive after 6 months. An analysis of the association between improvements in ENFD and monofilament testing found that the two tended to occur together, and this was significant $(p<0.05)$ for the right limb. Implications: Clinically important and statistically significant improvements in ENFD and monofilament sensation were associated with LMF-MC-PP in patients with DPN. When compared to the decrease in ENFD expected among DPN patients, the improvements are even more clinically sig-
\end{abstract}


nificant. These findings should be validated in a larger, placebo-controlled study.

\title{
Keywords
}

\author{
Diabetic Neuropathy, Epidermal Nerve Fiber Density, LMF-MC-PP, Monofilament Testing, \\ Small Nerve Fibers
}

\section{Introduction}

Diabetic sensorimotor peripheral neuropathy (DSPN) is prevalent among the population of patients with type 2 diabetes occurring in up to $50 \%$ of diabetic patients [1]-[6]. Factors associated with DSPN include age, duration of diabetes and severity of hyperglycemia [1] [6]-[9]. No treatments modify the underlying disease; existing treatment options focus only on symptomatic relief of pain. Thus, a need exists for effective treatments that modify the underlying disease state as well as improve symptoms [10]-[12].

L-methylfolate calcium is the biologically active form of folic acid and may have a role in improving endothelial function and correcting the underlying pathology of DPN [13]. The rationale for using LMF combined with vitamins B12 and B6 as potential disease modifying therapy is based on results from preclinical and clinical studies showing improved endothelial function, reduced oxidative stress and improvements in objective measures in animal models of neuropathy [14]-[21].

The combination of L-methylfolate calcium-methylcobalamin-pyridoxal-5-phospate (LMF-MC-PP, Metanx, Nestlé Health Science - Pamlab, Inc., Covington, LA) is a prescription medical food. Previous studies with LMF-MC-PP in patients with DPN have reported significant improvements in neuropathic pain, quality of life, as well as sensory perception as measured by 1-point (tactile) and 2-point (sensory discrimination) tests [22]-[27]. The largest study by Fonseca et al. demonstrated that LMF-MC-PP significantly improved neuropathic symptoms and quality of life in DPN patients but did not affect vibration perception threshold compared to placebo [22]. In a case series of 11 DPN patients treated with LMF-MC-PP who underwent repeat skin punch biopsies, treatment for 6 months with LMF-MC-PP was associated with a significant improvement in epidermal nerve fiber density (ENFD) [27].

The objective of this study was to evaluate the effects of LMF-MC-PP on ENFD and monofilament sensation in patients with diabetic neuropathy. Monofilament sensation testing has been shown to predict ulceration and amputation in patients with DPN [28]-[34] and is therefore recommended by the American Diabetes Association (ADA) and American Association of Clinical Endocrinologists (AACE) as a part of the assessment and management of diabetic neuropathy [35]-[37].

\section{Methods}

\subsection{Study Design}

This was an observational study of patients presenting to a community podiatry clinic with type 2 diabetes and clinical DPN who were selected to undergo skin biopsies to evaluate ENFD before and after treatment with LMF-MC-PP. The study protocol was reviewed by an institutional review board that granted a waiver of informed consent. All patient information was de-identified prior to collection.

\subsection{Patient Selection}

Patients with type 2 diabetes and diagnosed with diabetic peripheral polyneuropathy based on vibratory sensorium, warm-cold discrimination or loss of protective sensorium, and an ENFD assessment were included. Patients with a medical history or laboratory tests that documented chemotherapy, heavy metal poisoning, pernicious anemia, sero-positive or sero-negative arthropathy or any systemic metabolic inflammatory disease process other than cardiovascular disease were excluded from this study.

\subsection{Study Assessments}

A screening qualitative neurological examination was conducted using all of three methods. Abnormal vibratory 
sensorium - a $128 \mathrm{C}$ tuning fork was held to the dorso-medial bony eminence of the \#1 interphalangeal joint of each foot. When the patient reported that no vibration was any longer evident, the tuning fork was moved to the analogous articulation of the thumb and the response was noted. Abnormal warm-cold discrimination-separately, two different metals of known different heat capacities were applied in sequence to the plantar tuft of the Hallux. The patient was requested to identify which felt warmer and which felt colder. This technique was applied to the palm of the hand, in sequence, after the toe, to confirm the response of the difference between the two metals. Protective sensorium loss - an 11-point load test of $10 \mathrm{gm} / \mathrm{cm}^{2}$ was applied to the dorsal and plantar aspects of the feet and toes following the standard Semmes-Weinstein Monofilament (SWM) technique [37]. If any of these three qualitative neurological parameters were abnormal, the patient underwent an skin biopsy for ENFD quantitation.

ENFD analysis was conducted on each leg via a $3 \mathrm{~mm}$ punch biopsy of skin, $10 \mathrm{~cm}$ superior to the tip of the lateral malleolus, utilizing established protocols for atraumatic technique to quantify the extent of small nerve fiber loss. The patient was placed in a left lateral decubitus position. An impression in the skin was made in the lateral right ankle at a height of $10.5 \mathrm{~cm}$ superior to the lateral malleolus. Isopropyl alcohol was applied to the skin without rubbing, and topical ethyl chloride was applied to chill only the skin superior to the impression site prior to injection. The area superior to skin impression was atraumatically anesthetized with $2 \%$ lidocaine with epinephrine $1 \mathrm{cc}$ taking care to avoid the biopsy site. When adequate anesthesia was established, a sterile $3 \mathrm{~mm}$ skin punch was gently used to dissect the biopsy from the surrounding skin. Utilizing atraumatic forceps and iris scissors and without touching the skin in the process, the biopsy was removed and placed in a marked fixative vial. Between 6 and 24 hours later, the biopsy was removed from the fixative, rinsed, and placed into dry ice for shipping to Bako Pathology (Alpharetta, GA) following standard protocol and technique. A steri-strip adhesive bandage and antibiotic dressing was applied to the wound. The patient was placed in the right lateral decubitus position, and the procedure was repeated for the left ankle biopsy. The patient was advised verbally and in writing of the correct wound care protocol for the biopsy sites.

Upon receipt of the bilateral ENFD analysis results from Bako Pathology (usually within 2 weeks), the results and their clinical significance were discussed with the patient. An abnormal ENFD analysis was considered one in which the SNF count was lower than 7.1 fibers/mm and/or morphological degenerative changes were noted. If an abnormal ENFD analysis of either lower extremity was obtained, a prescription for LMF-MC-PP (L-methylfolate calcium $3 \mathrm{mg}$; pyridoxal 5'-phosphate $35 \mathrm{mg}$, methylcobalamin $2 \mathrm{mg}$ ) medical food one capsule twice daily was prescribed, and the patient was encouraged to persist with therapy. The assessment was performed 6 months after the initial analysis to determine the effect of LMF-MC-PP on ENFD. The Semmes-Weinstein Monofilament test was also repeated at 6 months.

\subsection{Statistical Analysis}

Descriptive statistics were calculated including means, standard deviations for continuous variables, and frequencies and percentages for categorical variables.

Two outcome measures were studied: 1) small nerve fiber density and 2) monofilament test results for sensation. These measures were evaluated at baseline (i.e. before the initiation of LMF-MC-PP therapy) and at 6 months post-baseline for both the right and left legs. For ENFD, the change in the number of fibers per millimeter was calculated, and paired t-tests were carried out to evaluate the significance. For monofilament test results, the presence/absence of sensation was analyzed. The Kappa coefficient and Bowker's test were used to evaluate the significance of change. The correlation of change from baseline to 6 months between ENFD and monofilament test was investigated with a Spearman's correlation coefficient. A significant result was considered $\mathrm{p}<$ 0.05 .

\section{Results}

A total of 123 diabetic patients were evaluated and treated between 2010 and 2014 and were included in the analysis. Monofilament testing results were available for all 123 patients and ENFD analysis results were available for 122. Baseline and 6-month data for ENFD results were available for 111 patients for the dominant limb analysis and 110 patients for the non-dominant limb assessment. Baseline demographic and clinical characteristics reveal a population that was consistent with patients having type 2 diabetes (Table 1). Two-thirds were at least 65 years of age, and all were treated with some type of hypoglycemic therapy. 
Table 1. Baseline characteristics.

\begin{tabular}{|c|c|}
\hline & N (\%) \\
\hline Baseline Year & 123 \\
\hline 2010 & $61(49.6)$ \\
\hline 2011 & $25(20.3)$ \\
\hline 2012 & $20(16.3)$ \\
\hline 2013 & $15(12.2)$ \\
\hline 2014 & $2(1.6)$ \\
\hline \multicolumn{2}{|l|}{ Age, years } \\
\hline$<65$ & $45(36.6)$ \\
\hline$\geq 65$ & $78(63.4)$ \\
\hline \multicolumn{2}{|l|}{ Gender } \\
\hline Female & $49(39.8)$ \\
\hline Male & $74(60.2)$ \\
\hline \multicolumn{2}{|l|}{ Ethnicity } \\
\hline Non-hispanic & $105(86.1)$ \\
\hline Hispanic or Latino & $17(13.9)$ \\
\hline \multicolumn{2}{|l|}{ Diabetes Treatment } \\
\hline Diet & $9(7.3)$ \\
\hline Metformin & $91(74.0)$ \\
\hline Insulin & $31(25.2)$ \\
\hline Other & $50(40.7)$ \\
\hline
\end{tabular}

\subsection{Epidermal Nerve Fiber Density Analysis}

A significant $(\mathrm{p}<0.05)$ improvement from baseline to 6 months was observed in the ENFD analysis for the right limb, left limb, dominant limb, and non-dominant limb (Table 2). The mean magnitude of change was at least 0.6 nerve fibers $/ \mathrm{mm}$ for the right limb and dominant limb but reached 1.1 nerve fibers $/ \mathrm{mm}$ for left limb.

\subsection{Monofilament Sensation Testing}

A significant $(\mathrm{p}=0.0001)$ improvement in monofilament sensation testing was observed with LMF-MC-PP treatment. Of 63 patients with absent sensation at baseline, $38(60.3 \%)$ had intact sensation after 6 months of treatment with LMF-MC-PP. In contrast, only three (5.0\%) of 60 patients with intact sensation at baseline lost sensation after 6 months of treatment (Table 3). Overall, 60 (48.8\%) of 123 patients had intact sensation at baseline, and $95(77.2 \%)$ had intact sensation at 6 months (Figure 1). Not more than three patients (depending upon which limb) experienced worsening sensation at six months.

\subsection{Correlation between ENFD and Monofilament Sensation Testing}

An analysis was conducted of the association between monofilament sensation improvement and ENFD improvement where the latter was defined as an increase from baseline of at least 1.0 fibers $/ \mathrm{mm}$. Among those with ENFD improvement, 33.3\% (left foot; $p=0.44$ ) and $44.2 \%$ (right foot; $p=0.02$ ) of patients also improved in sensation at six months (Table 4). When ENFD improvement was defined as 0.5 fibers $/ \mathrm{mm}$ (Table 5), then the respective percentages were $34.7 \%$ (left foot; $p=0.26$ ) and $41.7 \%$ (right foot; $p=0.03$ ). 


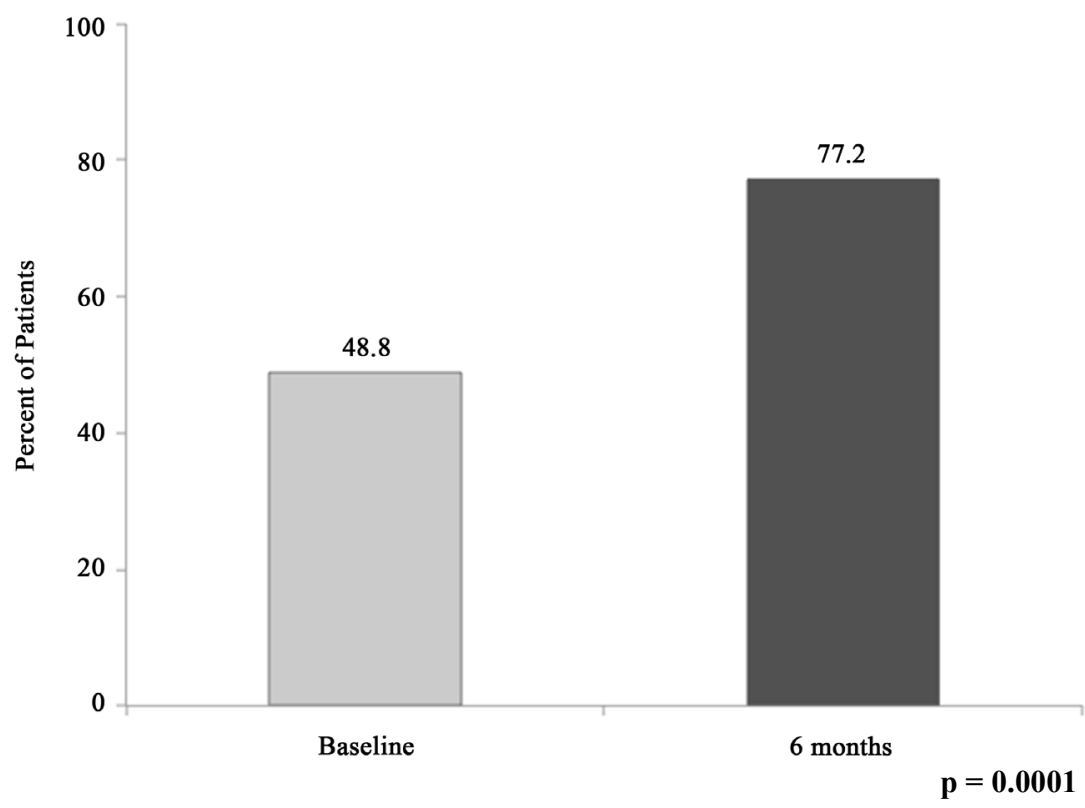

Figure 1. Patients with intact sensorium before and after LMF-MC-PP.

Table 2. Analysis of ENFD results at baseline, after 6 months, and change from baseline to 6 months.

\begin{tabular}{|c|c|c|c|}
\hline & $\mathrm{N}$ & Mean $\pm \mathrm{SD}$ (\# fibers/mm) & p-value ${ }^{*}$ \\
\hline Right Foot Baseline & 122 & $5.2 \pm 5.2$ & \\
\hline Right Foot 6 Month & 122 & $5.7 \pm 6.0$ & \\
\hline Right Foot Change from Baseline & 122 & $0.6 \pm 3.7$ & 0.0433 \\
\hline Left Foot Baseline & 123 & $4.7 \pm 4.4$ & \\
\hline Left Foot 6 Month & 122 & $5.7 \pm 5.8$ & \\
\hline Left Foot Change from Baseline & 122 & $1.1 \pm 3.4$ & 0.0004 \\
\hline Dominant Limb Baseline & 111 & $5.1 \pm 5.1$ & \\
\hline Dominant Limb 6 Month & 111 & $5.7 \pm 5.9$ & \\
\hline Dominant Limb Change from Baseline & 111 & $0.6 \pm 3.8$ & 0.0393 \\
\hline Non-dominant Limb Baseline & 111 & $4.8 \pm 4.4$ & \\
\hline Non-dominant Limb 6 Month & 110 & $5.9 \pm 5.8$ & \\
\hline Non-dominant Limb Change from Baseline & 110 & $1.0 \pm 3.4$ & 0.0012 \\
\hline
\end{tabular}

*One-sided test for improvement at 6-month follow-up.

Table 3. Monofilament test results.

\begin{tabular}{|c|c|c|c|c|c|}
\hline \multirow{2}{*}{ Baseline } & \multirow{2}{*}{6 Month } & \multirow{2}{*}{ Change at 6 month } & \multirow{2}{*}{$\mathrm{N}(\%)$} & Agreement Statistic & Symmetry of Disagreement \\
\hline & & & & Kappa Coefficient & Bowker's Test \\
\hline Negative & Negative & Negative to Negative & $25(20.3)$ & \multirow{4}{*}{$0.24, p=0.0001$} & \multirow{4}{*}{$\mathrm{p}=0.0001$} \\
\hline \multirow{3}{*}{ Positive } & Positive & Negative to Positive & $38(30.9)$ & & \\
\hline & Negative & Positive to Negative & $3(2.4)$ & & \\
\hline & Positive & Positive to Positive & $57(46.3)$ & & \\
\hline
\end{tabular}


Table 4. Correlation between SNF and monofilament testing. Proportion of patients with type 2 diabetes with improvement of at least $1.0 \mathrm{~mm} /$ fiber from baseline.

\begin{tabular}{|c|c|c|c|c|c|c|}
\hline & \multicolumn{4}{|c|}{$10 \mathrm{~g}$ Changes } & & \\
\hline & \multirow{2}{*}{$\begin{array}{c}\text { Improved } \\
\mathrm{N}(\%)\end{array}$} & \multirow{2}{*}{$\begin{array}{c}\text { No Change } \\
\text { N (\%) }\end{array}$} & \multirow{2}{*}{$\begin{array}{c}\text { Worsened } \\
\mathrm{N}(\%)\end{array}$} & \multirow{2}{*}{$\begin{array}{c}\text { Total } \\
\mathrm{N}\end{array}$} & \multicolumn{2}{|c|}{ Measures of Association } \\
\hline & & & & & Spear-man's & p-value \\
\hline \multicolumn{5}{|c|}{ Right foot difference from baseline } & 0.219 & 0.0156 \\
\hline$\geq 1$ Improvement & $19(44.2)$ & $24(55.8)$ & $0(0.0)$ & 43 & & \\
\hline$<1$ Improvement & $19(24.1)$ & $58(73.4)$ & $2(2.5)$ & 79 & & \\
\hline \multirow[t]{2}{*}{ Total } & $38(31.2)$ & $82(67.2)$ & $2(1.6)$ & 122 & & \\
\hline & \multicolumn{3}{|c|}{ Left foot difference from baseline } & & 0.071 & 0.4352 \\
\hline$\geq 1$ Improvement & $14(33.3)$ & $28(66.7)$ & $0(0.0)$ & 42 & & \\
\hline$<1$ Improvement & $23(28.8)$ & $54(67.5)$ & $3(3.8)$ & 80 & & \\
\hline \multirow[t]{2}{*}{ Total } & $37(30.3)$ & $82(67.2)$ & $3(2.5)$ & 122 & & \\
\hline & \multicolumn{3}{|c|}{ Dominant limb difference from baseline } & & 0.149 & 0.1180 \\
\hline$\geq 1$ Improvement & $17(40.5)$ & $25(59.5)$ & $0(0.0)$ & 42 & & \\
\hline$<1$ Improvement & $19(27.5)$ & $48(69.6)$ & $2(2.9)$ & 69 & & \\
\hline \multirow[t]{2}{*}{ Total } & $36(32.4)$ & $73(65.8)$ & $2(1.8)$ & 111 & & \\
\hline & \multicolumn{3}{|c|}{ Non-dominant limb difference from baseline } & & 0.107 & 0.2647 \\
\hline$\geq 1$ Improvement & $14(37.8)$ & $23(62.2)$ & $0(0.0)$ & 37 & & \\
\hline$<1$ Improvement & $21(28.8)$ & $50(68.5)$ & $2(2.7)$ & 73 & & \\
\hline Total & $35(31.8)$ & $73(66.4)$ & $2(1.8)$ & 110 & & \\
\hline
\end{tabular}

Table 5. Correlation between SNF and monofilament testing. Proportion of patients with type 2 diabetes with improvement of at least $0.5 \mathrm{~mm} /$ fiber from baseline.

\begin{tabular}{|c|c|c|c|c|c|c|}
\hline \multicolumn{7}{|c|}{$10 \mathrm{~g}$ Changes } \\
\hline & \multirow{2}{*}{$\begin{array}{c}\text { Improved } \\
\mathrm{N}(\%)\end{array}$} & \multirow{2}{*}{$\begin{array}{c}\text { No Change } \\
\text { N }(\%)\end{array}$} & \multirow{2}{*}{$\begin{array}{c}\text { Worsened } \\
\text { N (\%) }\end{array}$} & \multirow{2}{*}{$\begin{array}{c}\text { Total } \\
\mathrm{N}\end{array}$} & \multicolumn{2}{|c|}{ Measures of Association } \\
\hline & & & & & Spearman's & p-value \\
\hline \multicolumn{5}{|c|}{ Right foot difference from baseline } & 0.1961 & 0.0304 \\
\hline$\geq 0.5$ Improvement & $20(41.7)$ & $28(58.3)$ & $0(0.0)$ & 48 & & \\
\hline$<0.5$ Improvement & $18(24.3)$ & $54(73.0)$ & $2(2.7)$ & 74 & & \\
\hline \multirow[t]{2}{*}{ Total } & $38(31.2)$ & $82(67.2)$ & $2(1.6)$ & 122 & & \\
\hline & \multicolumn{3}{|c|}{ Left foot difference baseline } & & 0.1037 & 0.2559 \\
\hline$\geq 0.5$ Improvement & $17(34.7)$ & $32(65.3)$ & $0(0.0)$ & 42 & & \\
\hline$<0.5$ Improvement & $20(27.4)$ & $50(68.5)$ & $3(4.1)$ & 80 & & \\
\hline \multirow[t]{2}{*}{ Total } & $37(30.3)$ & $82(67.2)$ & $3(2.5)$ & 122 & & \\
\hline & \multicolumn{3}{|c|}{ Dominant limb difference from baseline } & & 0.1376 & 0.1499 \\
\hline$\geq 0.5$ Improvement & $18(39.1)$ & $28(60.9)$ & $0(0.0)$ & 46 & & \\
\hline$<0.5$ Improvement & $18(27.7)$ & $45(69.2)$ & $2(3.1)$ & 65 & & \\
\hline \multirow[t]{2}{*}{ Total } & $36(32.4)$ & $73(65.8)$ & $2(1.8)$ & 111 & & \\
\hline & \multicolumn{4}{|c|}{ Non-dominant limb difference from baseline } & 0.1243 & 0.1956 \\
\hline$\geq 0.5$ Improvement & $17(37.8)$ & $28(62.2)$ & $0(0.0)$ & 45 & & \\
\hline$<0.5$ Improvement & $18(27.7)$ & $45(69.2)$ & $2(3.1)$ & 65 & & \\
\hline Total & $35(31.8)$ & $73(66.4)$ & $2(1.8)$ & 110 & & \\
\hline
\end{tabular}




\section{Discussion}

The results from this analysis confirm and extend previous findings with LMF-MC-PP in patients with diabetic neuropathy [22]-[24]. Importantly, results from this study demonstrated improvement over time in lower limb ENFD and by limb sensation assessed by the Semmes-Weinstein monofilament test. The mean improvement in ENFD over 6 months ranged from 0.6 to 1.1 fibers $/ \mathrm{mm}$. This improvement is smaller in comparison to the increase of 1.5 fibers $/ \mathrm{mm}$ observed by Jacobs and Cheng; [27] however, that population was small $(\mathrm{N}=11)$ with a lower baseline ENFD (mean 1.6 fibers/mm). The findings of the present study are likely representative of expected results with LMF-MC-PP in a population with less nerve degeneration at baseline. In a parallel study of 116 DPN patients followed for 2 years, the rate of ENFD change over time without intervention was estimated at -0.68 fibers $/ \mathrm{mm} /$ year (Vinik, data on file).

The positive correlation between the objective ENFD measurement and the clinical sensation test, albeit statistically significant only in the right foot, reinforces the strength of the results through demonstrating that improvement on both features is happening in the same patient.

The rationale for the use of LMF-MC-PP in this population of patients with confirmed diabetic neuropathy is based on previously reported studies. In animal studies, the components of LMF-MC-PP have been shown to improve both endothelial function and nerve conduction [13]-[16] [18]. Results from smaller open-label studies of LMF-MC-PP in patients with diabetic neuropathy reported improvements in sensation, ENFD, and symptoms [23] [25]. In a prospective, double-blind, randomized study, the effects of LMF-MC-PP in 214 patients with diabetic neuropathy were evaluated [22]. After 24 weeks, a significant improvement in neuropathy symptoms was observed together with improvements in quality of life.

Limitations of this study include its observational design and lack of a parallel control group. However, the objective nature of the biopsy findings from ENFD, as the primary endpoint, adds credibility to the results. In addition, these findings are limited by the 6-month duration of follow up. Longer term studies are needed to more fully assess the effects of LMF-MC-PP on a slowly progressing disease such as diabetic neuropathy. No information on adverse events was systematically collected, but no serious adverse events were noted. In a controlled study of patients with type 2 diabetes and peripheral neuropathy, LMF-MC-PP was well tolerated with a low incidence of adverse events that were not significantly different from adverse events reported with placebo [22].

\section{Conclusion}

These results build on previous findings in patients with diabetic neuropathy that LMF-MC-PP provides improvement in objective and subjective measures of neuropathy. Larger, controlled studies evaluating LMFMC-PP in patients with neuropathy that utilize objective measures of nerve function are warranted to confirm these findings and further establish the benefits of this therapy in patients with DPN.

\section{Acknowledgements}

The authors acknowledge the editorial assistance of Richard S. Perry, Pharm D in the preparation of this manuscript, which was supported by Nestlé Health Science-Pamlab, Inc., Covington, Louisiana.

\section{Conflict of Interest}

This work was supported by grants from Nestlé Health Science-Pamlab, Inc, Covington, Louisiana. The sponsor participated in the study design, analysis, and review of the manuscript but was not involved in data collection.

Mrs. Barrentine is a full-time employee of Nestle Health Science-Pamlab, Inc. Research support for this study was provided to Dr. McNamara; he has no other conflicts of interest. Dr. De Vol received research support for this study; he has no other conflicts of interest. Dr. Vinik is a board member of Medscape. He serves as a consultant for Pfizer, Merck, Nestlé Health Science-Pamlab, Inc, Hydra Biosciences, Neurometrix, and ISIS Pharmaceuticals. Dr. Vinik has received research support from Pfizer, Impeto Medical Daiichi Sankyo Pharma, Tercica, Viromed, Intarcia Therapeutics, Vero Science, and Novo Nordisk. He also has received grants from the American Diabetes Association and from the National Institutes of Health. He is on the speaker's bureau for Merck and Nestlé Health Science-Pamlab, Inc. 


\section{References}

[1] Davies, M., Brophy, S., Williams, R. and Taylor, A. (2006) The Prevalence, Severity, and Impact of Painful Diabetic Peripheral Neuropathy in Type 2 Diabetes. Diabetes Care, 29, 1518-1522. http://dx.doi.org/10.2337/dc05-2228

[2] Feldman, E.L. and Vincent, A. (2004) The Prevalence, Impact, and Multifactorial Pathogenesis of Diabetic Peripheral Neuropathy. Advanced Studies in Medicine, 4, S642-S649.

[3] Gore, M., Brandenburg, N.A., Hoffman, D.L., Tai, K.S. and Stacey, B. (2006) Burden of Illness in Painful Diabetic Peripheral Neuropathy: The Patients' Perspectives. Journal of Pain, 7, 892-900. http://dx.doi.org/10.1016/j.jpain.2006.04.013

[4] Sadosky, A., Hopper, J. and Parsons, B. (2014) Painful Diabetic Peripheral Neuropathy: Results of a Survey Characterizing the Perspectives and Misperceptions of Patients and Healthcare Practitioners. Patient, 7, 107-114. http://dx.doi.org/10.1007/s40271-013-0038-8

[5] Sadosky, A., Schaefer, C., Mann, R., Bergstrom, F., Baik, R., Parsons, B., Nalamachu, S., Nieshoff, E., Stacey, B.R., Anschel, A. and Tuchman, M. (2013) Burden of Illness Associated with Painful Diabetic Peripheral Neuropathy among Adults Seeking Treatment in the US: Results from a Retrospective Chart Review and Cross-Sectional Survey. Journal of Diabetes, Metabolic Syndrome and Obesity, 6, 79-92. http://dx.doi.org/10.2147/DMSO.S37415

[6] Tesfaye, S. (2004) Epidemiology and Etiology of Diabetic Peripheral Neuropathies. Advanced Studies in Medicine, 4, S1014-S1021.

[7] Diabetic Neuropathies: The Nerve Damage of Diabetes (2009) Bethesda (MD): US Department of Health and Human Services. Report No.: NIH Publication No. 09-3185.

http://diabetes.niddk.nih.gov/dm/pubs/neuropathies/neuropathies.pdf

[8] Boulton, A.J.M. (2005) Management of Diabetic Peripheral Neuropathy. Clinical Diabetes, 23, 9-15. http://dx.doi.org/10.2337/diaclin.23.1.9

[9] Tölle, T., Xu, X. and Sadosky, A.B. (2006) Painful Diabetic Neuropathy: A Cross-Sectional Survey of Health State Impairment and Treatment Patterns. Journal of Diabetes and Its Complications, 20, 26-33. http://dx.doi.org/10.1016/j.jdiacomp.2005.09.007

[10] Head, K.A. (2005) Peripheral Neuropathy: Pathogenic Mechanisms and Alternative Therapies. Alternative Medicine Review, 11, 294-329.

[11] Ziegler, D. (2009) Painful Diabetic Neuropathy: Advantage of Novel Drugs over Old Drugs? Diabetes Care, 32, S414S419.

[12] Miranda-Massari, J.R., Gonzalez, M.J., Jimenez, F.J., Allende-Vigo, M.Z. and Duconge, J. (2011) Metabolic Correction in the Management of Diabetic Peripheral Neuropathy: Improving Clinical Results beyond Symptom Control. Current Clinical Pharmacology, 6, 260-273. http://dx.doi.org/10.2174/157488411798375967

[13] van Etten, R.W., de Koning, E.J., Verhaar, M.C., Gaillard, C.A. and Rabelink, T.J. (2002) Impaired NO-Dependent Vasodilation in Patients with Type II (Non-Insulin-Dependent) Diabetes Mellitus Is Restored by Acute Administration of Folate. Diabetologia, 45, 1004-1010. http://dx.doi.org/10.1007/s00125-002-0862-1

[14] Li, J.-B., Wang, C.-Y., Chen, J.-W., Li, X.-L., Feng, Z.-G. and Ma, H.-T. (2010) The Preventive Efficacy of Methylcobalamin on Rat Peripheral Neuropathy Influenced by Diabetes via Neural IGF-1 Levels. Nutritional Neuroscience, 13, 79-86. http://dx.doi.org/10.1179/147683010X12611460763607

[15] Jain, S.K. and Lim, G. (2001) Pyridoxine and Pyridoxamine Inhibits Superoxide Radicals and Prevents Lipid Peroxidation, Protein Glycosylation, and $\left(\mathrm{Na}^{+}+\mathrm{K}^{+}\right)$-ATPase Activity Reduction in High Glucose-Treated Human Erythrocytes. Free Radical Biology and Medicine, 30, 232-237. http://dx.doi.org/10.1016/S0891-5849(00)00462-7

[16] Obrosova, I. and Shevalye, H. (2011) Metanx Alleviates Diabetes-Induced Large and Small Fiber Neuropathies and Promotes Small Sensory Nerve Fiber Regeneration. Diabetes, 60, 652-P.

[17] Sandireddy, R., Yerra, V.G., Areti, A., Komirishetty, P. and Kumar, A. (2014) Neuroinflammation and Oxidative Stress in Diabetic Neuropathy: Futuristic Strategies Based on These Targets. International Journal of Endocrinology, 2014, Article ID: 674987. http://dx.doi.org/10.1155/2014/674987

[18] Shevalye, H., Watcho, P., Stavniichuk, R., Dyukova, E., Lupachyk, S. and Obrosova, I.G. (2012) Metanx Alleviates Multiple Manifestations of Peripheral Neuropathy and Increases Intraepidermal Nerve Fiber Density in Zucker Diabetic Fatty Rats. Diabetes, 61, 2126-2133. http://dx.doi.org/10.2337/db11-1524

[19] Yilmaz, M., Aktug, H., Oltulu, F. and Erbas, O. (2013) Neuroprotective Effects of Folic Acid on Experimental Diabetic Peripheral Neuropathy. Toxicology and Industrial Health.[Epub ahead of print]

[20] Nakamura, S., Li, H., Adijiang, A., Pischetsrieder, M. and Niwa, T. (2007) Pyridoxal Phosphate Prevents Progression of Diabetic Nephropathy. Nephrology Dialysis Transplantation, 22, 2165-2174. http://dx.doi.org/10.1093/ndt/gfm166

[21] Yaqub, B.A., Siddique, A. and Sulimani, R. (1992) Effects of Methylcobalamin on Diabetic Neuropathy. Clinical 
Neurology and Neurosurgery, 94, 105-111. http://dx.doi.org/10.1016/0303-8467(92)90066-C

[22] Fonseca, V.A., Lavery, L.A., Thethi, T.K., et al. (2013) Metanx in Type 2 Diabetes with Peripheral Neuropathy: A Randomized Trial. The American Journal of Medicine, 126, 141-149. http://dx.doi.org/10.1016/j.amjmed.2012.06.022

[23] Jacobs, A.M. and Cheng, D. (2013) Addition of Metanx in Pregabalin Partial Responders for Painful Diabetic Neuropathy. Journal of Diabetes Mellitus, 3, 134-138. http://dx.doi.org/10.4236/jdm.2013.33020

[24] Trippe, B.S., Barrentine, L.W., Curole, M.V. and Tipa, E. (2016) Nutritional Management of Patients with Diabetic Peripheral Neuropathy with Metanx: Results of a Real-World Patient Experience Trial. Current Medical Research and Opinion, 32, 219-227. http://dx.doi.org/10.1185/03007995.2015.1103215

[25] Walker, M.J., Morris, L.M. and Cheng, D. (2010) Improvement of Cutaneous Sensitivity in Diabetic Peripheral Neuropathy with Combination L-Methylfolate, Methylcobalamin, and Pyridoxal 5-Phosphate. Reviews in Neurological Diseases, 7, 132-139.

[26] Vinik, A. (2013) A Medicinal Food Provides Food for Thought in Managing Diabetic Neuropathy. The American Journal of Medicine, 126, 95-96. http://dx.doi.org/10.1016/j.amjmed.2012.08.008

[27] Jacobs, A.M. and Cheng, D. (2011) Management of Diabetic Small-Fiber Neuropathy with Combination L-Methylfolate, Methylcobalamin, and Pyridoxal 5'-Phosphate. Reviews in Neurological Diseases, 8, 39-47.

[28] Armstrong, D.G., Lavery, L.A., Vela, S.A., Quebedeaux, T.L. and Fleischli, J.G. (1998) Choosing a Practical Screening Instrument to Identify Patients at Risk for Diabetic Foot Ulceration. Archives of Internal Medicine, 158, $289-292$. http://dx.doi.org/10.1001/archinte.158.3.289

[29] Armstrong, D.G. and Lavery, L.A. (1998) Diabetic Foot Ulcers: Prevention, Diagnosis and Classification. American Family Physician, 57, 1325-1332, 1337-1338.

[30] Boulton, A.J. (1998) Lowering the Risk of Neuropathy, Foot Ulcers and Amputations. Diabetic Medicine, 15, S57-S59. http://dx.doi.org/10.1002/(SICI)1096-9136(1998120)15:4+<S57::AID-DIA741>3.3.CO;2-4

[31] Mayfield, J.A. and Sugarman, J.R. (2000) The Use of the Semmes-Weinstein Monofilament and Other Threshold Tests for Preventing Foot Ulceration and Amputation in Persons with Diabetes. The Journal of Family Practice, 49, S17S29.

[32] Pham, H., Armstrong, D.G., Harvey, C., Harkless, L.B., Giurini, J.M. and Veves, A. (2000) Screening Techniques to Identify People at High Risk for Diabetic Foot Ulceration: A Prospective Multicenter Trial. Diabetes Care, 23, 606611. http://dx.doi.org/10.2337/diacare.23.5.606

[33] Vinik, A.I., Nevoret, M., Casellini, C. and Parson, H. (2013) Neurovascular Function and Sudorimetry in Health and Disease. Current Diabetes Reports, 13, 517-532. http://dx.doi.org/10.1007/s11892-013-0392-x

[34] Vinik, A.I., Nevoret, M.L. and Casellini, C. (2015) The New Age of Sudomotor Function Testing: A Sensitive and Specific Biomarker for Diagnosis, Estimation of Severity, Monitoring Progression, and Regression in Response to Intervention. Frontiers in Endocrinology, 6, 94. http://dx.doi.org/10.3389/fendo.2015.00094

[35] Boulton, A.J., Armstrong, D.G., Albert, S.F., Frykberg, R.G., Hellman, R., Kirkman, M.S., et al. (2008) Comprehensive Foot Examination and Risk Assessment. A Report of the Task Force of the Foot Care Interest Group of the American Diabetes Association, with Endorsement by the American Association of Clinical Endocrinologists. Physical Therapy, 88, 1436-1443.

[36] Handelsman, Y., Bloomgarden, Z.T., Grunberger, G., Umpierrez, G., Zimmerman, R.S., Bailey, T.S., et al. (2015) American Association of Clinical Endocrinologists and American College of Endocrinology - Clinical Practice Guidelines for Developing a Diabetes Mellitus Comprehensive Care Plan-2015. Endocrine Practice, 21, 1-87. http://dx.doi.org/10.4158/EP15672.GLSUPPL

[37] Kumar, S., Fernando, D.J., Veves, A., Knowles, E.A., Young, M.J. and Boulton, A.J. (1991) Semmes-Weinstein Monofilaments: A Simple, Effective and Inexpensive Screening Device for Identifying Diabetic Patients at Risk of Foot Ulceration. Diabetes Research and Clinical Practice, 13, 63-67. http://dx.doi.org/10.1016/0168-8227(91)90034-B 